\title{
Potentiation of a functional autoantibody in narcolepsy by a cholinesterase inhibitor
}

\author{
Michael W Jackson ${ }^{1}$, Nicolas J Spencer ${ }^{2}$, Joanne H Reed ${ }^{1}$, Anthony JF Smith ${ }^{1}$ and Tom P Gordon ${ }^{1}$
}

We have recently reported the presence of an immunoglobulin $\mathrm{G}(\mathrm{lgG})$ autoantibody $(\mathrm{Ab})$ in patients with narcolepsy with cataplexy that abolishes spontaneous colonic migrating motor complexes (CMMCs) and increases smooth muscle tension and atropine-sensitive phasic contractions in a physiological assay of an isolated colon. In this study, we used the cholinesterase inhibitor, neostigmine, to explore the mechanism of the narcoleptic lgG-mediated disruption of enteric motor function in four patients with narcolepsy with cataplexy and to identify a pharmacological mimic of the Ab. Neostigmine potentiated the narcoleptic IgG-mediated increase in smooth muscle resting tension and phasic smooth muscle contractions by an atropine-sensitive mechanism but exerted no effect on resting tension in the presence of control IgG. Decreased frequency of CMMCs mediated by lgG with anti-M3R activity was reversed by neostigmine. Therefore, a challenge with a cholinesterase inhibitor improves the specificity of the CMMC assay for narcoleptic IgG. Tetrodotoxin (TTX), a neuronal sodium channel blocker, also abolished CMMCs and increased resting tone, and a similar potentiation was observed with neostigmine; thus, TTX is a mimic of the functional effects of the narcoleptic lgG in this bioassay. These findings provide a link to pharmacological studies of canine narcolepsy and are consistent with a functional blockade of both excitatory and inhibitory motor neurons by the narcoleptic Ab, similar to the TTX mimic, presumably by binding to an autoantigenic target expressed in both populations of neurons.

Laboratory Investigation (2009) 89, 1332-1339; doi:10.1038/labinvest.2009.108; published online 5 October 2009

KEYWORDS: narcolepsy; neostigmine; autoantibody; autoimmunity; neuron; myenteric

Human narcolepsy is a serious sleep disorder caused by excessive daytime sleepiness, cataplexy and other abnormal manifestations of rapid eye movement (REM) sleep. ${ }^{1,2}$ In humans, narcolepsy with cataplexy is characterised by reduced or absent hypocretin (orexin) levels in cerebrospinal fluid and by the selective loss of hypocretin-producing cells in the lateral hypothalamus. ${ }^{3-5}$ Although narcolepsy seems to be caused by deficiencies in hypocretin neurotransmission, the primary cause of the disease remains unknown. Although there is strong circumstantial evidence for an autoimmune basis for human narcolepsy, foremost being the close association of narcolepsy with the HLA-DQB1 0602 allele and the recent association with the T-cell receptor $\alpha$-locus, ${ }^{6-8}$ numerous attempts to identify specific antigenic targets of either a B- or T-cell response have proved unsuccessful (for review, see Black'; Scammell ${ }^{10}$; and Overeem et al ${ }^{11}$ ). More recently, hypothalamic gene expression profiling has identified a downregulation of insulin-like growth factor binding protein factor 3 in hypocretin neurons in both human narcolepsy and narcolepsy mouse models, suggesting that this might be a possible target, but immunoblot analysis of sera from narcolepsy subjects failed to identify an autoantibody (Ab) against this factor. ${ }^{12}$ Another pathogenic candidate identified in a genome-wide association study was the enzyme choline kinase- $\beta$, which is involved in the metabolism of choline, a precursor of the neurotransmitter, acetylcholine. ${ }^{13}$ Cholinergic mechanisms are important in REM sleep, and pharmacological studies have implicated acetylcholine in canine cataplexy ${ }^{14}$ and have suggested that a hypersensitivity of the muscarinic cholinergic system exists in the cataplectic dog brain. ${ }^{15}$

We have pioneered the use of whole-organ physiological assays to detect effects of Abs on different activation and inactivation states of ion channels and receptors that are amenable to pharmacological dissection. These functional Abs may fail to be detected by less-sensitive immunological

\footnotetext{
${ }^{1}$ Autoimmunity Research Laboratory, Department of Immunology, Allergy and Arthritis, Flinders Medical Centre and Flinders University, Adelaide, South Australia, Australia and ${ }^{2}$ Center for Neuroscience, Department of Human Physiology, Flinders Medical Centre and Flinders University, Adelaide, South Australia, Australia Correspondence: Dr MW Jackson, Department of Immunology, Allergy and Arthritis, Flinders University, GPO Box 2100, Adelaide, South Australia 5001, Australia. E-mail: michael.jackson@flinders.edu.au
} 
methods, such as immunofluorescence and immunoblotting. ${ }^{16-20}$ Recently, using a real-time bioassay of spontaneous, neuronally mediated colonic migrating motor complexes (CMMCs), we reported the presence of an immunoglobulin $\mathrm{G}(\mathrm{IgG}) \mathrm{Ab}$ in patients with narcolepsy with cataplexy that abolishes CMMC activity and increases resting tension and atropine-sensitive phasic contractions in colonic smooth muscle, suggesting the emergence of cholinergic responses secondary to a loss of smooth muscle inhibitory tone. We hypothesised that the putative narcoleptic $\mathrm{Ab}$ binds to an ion channel or receptor expressed on enteric neurons. ${ }^{21}$

In this study, we used the cholinesterase inhibitor, neostigmine, to further explore the mechanism by which narcoleptic IgG alters enteric nerve activity in the colon. We also examined the interaction between neostigmine and TTX, a neurotoxin that specifically binds to voltage-gated sodium channels and prevents action potential generation and propagation. ${ }^{22}$ This toxin abolishes CMMCs generated by enteric motor nerve activity. ${ }^{23}$ We found that TTX mimicked the functional effects of narcoleptic IgG in this isolated organ preparation. Our results are consistent with a model in which an $\mathrm{Ab}$ in patients with narcolepsy blocks neurotransmission of both excitatory and inhibitory enteric motor nerves.

\section{MATERIALS AND METHODS}

\section{Preparation of IgG and Antibody Fragments}

Total IgG was purified from blood collected from four patients with narcolepsy recruited from the Adelaide Institute for Sleep Health, Repatriation General Hospital, South Australia and from six healthy controls. Patients with narcolepsy (two men and two women) met the revised International Classification of Sleep Disorders criteria for the diagnosis of narcolepsy with cataplexy. Their mean age was 49.0 years (range: 21-83) and the duration of illness was 24.5 years (range: 6-41). All were positive for HLA-DR2/DQB1 ${ }^{\star} 0602$; had marked daytime sleepiness and unequivocal cataplexy; and had abnormal REM onset sleep confirmed by Multiple Sleep Latency Testing. IgG from each of the patients with narcolepsy with cataplexy was previously shown to abolish CMMC activity in the isolated colon assay. ${ }^{21}$ Neither narcoleptic or control subjects had conventional Abs on routine serological testing, including anti-nuclear antibody, antidsDNA, rheumatoid factor, anti-mitochondrial, anti-parietal cell or anti-thyroid microsomal antibodies. In addition, serum was obtained from a patient with primary Sjogren's syndrome (SS) whose $\operatorname{IgG}$ reduces the frequency of CMMCs. ${ }^{24}$ The study was approved by the Clinical Ethics Committee of the Flinders Medical Centre.

IgG was prepared using the caprylic acid precipitation technique $^{25}$ as described previously. ${ }^{21}$ Briefly, human sera was lowered to $\mathrm{pH} 4.8$ using $1 \mathrm{M}$ acetic acid and stirred vigorously while adding caprylic acid (yielding a final ratio of $0.74: 10$ ). The caprylic acid/serum mixture was then stirred for $30 \mathrm{~min}$ at room temperature, and centrifuged at $10000 \times g$ for $15 \mathrm{~min}$. The supernatant was recovered and filtered through a Millipore $0.25-\mu \mathrm{m}$ micron filter (Millipore Corporation, Billerica, MA, USA) and the $\mathrm{pH}$ was adjusted to 7.4 with $0.5 \mathrm{M}$ sodium hydroxide. Purified $\operatorname{IgG}$ was then dialysed against PBS pH 7.4 and concentrated to $40 \mathrm{mg} / \mathrm{ml}$ using a Millipore B15 concentrator (Millipore Corporation), and dialysed against Krebs solution ((mmol/l): $\mathrm{NaCl} 118, \mathrm{KCl}$ 4.7, $\mathrm{KH}_{2} \mathrm{PO}_{4} 1.2, \mathrm{MgSO}_{4}, 1.5, \mathrm{NaHCO}_{3} 25.0$, D-glucose 11.0 and $\mathrm{CaCl}_{2}$ 2.5). IgG was also purified from some patient serum using Protein G column chromatography (Amersham, Piscataway, NJ, USA) as described previously. ${ }^{16}$ IgG concentrations were determined by nephelometry (ICS II; Beckman, Brea, CA, USA). Monovalent Fab fragments were produced from some patient's IgG after a 4-h incubation at $37^{\circ} \mathrm{C}$ with papain $(0.8 / 100 \mathrm{mg}$ IgG; Sigma, Sydney, Australia), as published previously. ${ }^{26}$

\section{Functional Assay of Spontaneous, Colonic Migrating Motor Complexes in the Intact Colon}

CMMCs were studied as described previously. ${ }^{21}$ Male Balb/C mice $(20-30 \mathrm{~g})$ aged 10 to 12 weeks were housed in separate cages on a 12-h light-dark cycle for at least $72 \mathrm{~h}$ before the harvesting of colons. Mice were killed in the first $2 \mathrm{~h}$ of the light cycle and the colons were excised and suspended in a 100-ml organ bath containing Krebs' solution gassed with $95 \% \mathrm{O}_{2} / 5 \% \mathrm{CO}_{2}, \mathrm{pH} 7.4$ at $37^{\circ} \mathrm{C}$. Any faecal matter was gently flushed from the colon and the mesentery was dissected free. The mechanical activity of the circular smooth muscle was recorded using three force-displacement transducers (ADInstruments) attached at $2-\mathrm{cm}$ intervals to the mesenteric border of the colon under an initial tension of $6 \mathrm{mN}$. Transducer output was fed into a Quad bridge amplifier (ADInstruments) and was recorded using Chart v4.2 software and a PowerLab/8s data acquisition system (ADInstruments).

\section{Experimental Protocol and Data Analysis}

After a 60 -min equilibration period, patient or control $\mathrm{IgG}$ was added to yield a final concentration of $0.4 \mathrm{mg} / \mathrm{ml}$. After a 30-min incubation, circular smooth muscle resting tension (defined as the amplitude of contraction of the circular smooth muscle either between CMMCs, where present, or in the absence of CMMCs) was recorded at each of the three recording points for $30 \mathrm{~s}$ at 3-min intervals for a period of 15 min using the peak parameters-peak amplitude function of the DataPad on Chart v4.2 software (ADInstruments). The values were averaged to determine the mean contraction amplitude for each of the three recording points. Neostigmine (Sigma, St Louis, MO, USA) at a concentration of $1 \mu \mathrm{mol} / \mathrm{l}$ was then added to the preparations and incubated for $10 \mathrm{~min}$. The mean contraction amplitudes of the circular smooth muscle were again determined as described above. In some experiments, $0.6 \mu \mathrm{mol} / 1$ tetrodotoxin (TTX, Alomone Labs, Jerusalem, Israel) was used instead of IgG. In addition, in some experiments, $1-10 \mu \mathrm{mol} / \mathrm{l}$ atropine (Sigma) was added to the colon preparations in the presence of $\operatorname{IgG}$ and 
neostigmine, and the mean contraction amplitudes of the circular smooth muscle was determined as mentioned above. For comparisons between the pre- and post-drug circular smooth muscle resting tension, the mean contraction amplitudes at the three recording points were compared by the Wilcoxon matched-pairs nonparametric test using GraphPad Prism (version 5.0.1 for Macintosh; GraphPad Software, San Diego, CA, USA).

\section{RESULTS}

Neostigmine Potentiates the Narcoleptic IgG-Mediated Increase in Colonic Smooth Muscle Resting Tension and Spontaneous, Phasic Contractions

We have previously reported that addition of IgG from patients with narcolepsy-cataplexy profoundly disrupts the CMMC activity of an isolated mouse colon; however, the mechanisms of action of this IgG was unclear. ${ }^{21}$ As CMMCs are predominantly mediated by the release of acetylcholine from cholinergic motor neurons, ${ }^{27}$ we used the cholinesterase inhibitor, neostigmine, to further define the mechanisms by which narcoleptic IgG mediates colonic dysfunction. In the isolated mouse colon, spontaneous, regular CMMCs were recorded as contractions mediated by excitatory motor activity migrating from the proximal to distal colon at intervals of 3-5 min, separated by periods of quiescence during which inhibitory motor activity regulates smooth muscle tone. Addition of six IgG preparations $(0.4 \mathrm{mg} / \mathrm{ml})$ purified from four patients with narcolepsy-cataplexy to individual colon preparations resulted in the abrogation of CMMCs with an increase in smooth muscle resting tension and spontaneous, phasic contractions in all cases. ${ }^{21}$ Subsequent addition of neostigmine $(1 \mu \mathrm{mol} / \mathrm{l})$ to the water bath significantly increased smooth muscle resting tension $(P<0.0001$, Wilcoxon matched-pairs comparison of pre- $v s$ post-drug mean contraction amplitude), and increased the amplitude of spontaneous, phasic contractions but did not restore CMMC activity (Figure $1 \mathrm{a}, \mathrm{b}$ and e). Similar results were obtained after the addition of neostigmine to a preparation incubated with narcoleptic IgG purified by a Protein G column (in which IgM and IgA were undetectable by nephelometry) verifying that the results are independent of the method of IgG preparation (Supplementary Figure 1). Neostigmine did not significantly alter the smooth muscle resting tension in experiments using normal control IgGs $(P=0.076, n=6)$ (Figure $1 \mathrm{c}$ and $\mathrm{e}$ ). To determine whether neostigmine reversed nonspecific IgG-induced suppression of CMMCs, the concentration of control IgG in the organ bath was doubled to $0.8 \mathrm{mg} / \mathrm{ml}$, which produced a nonspecific quenching of CMMCs. Adding $1 \mu \mathrm{mol}$ neostigmine to these preparations restored CMMC activity without altering smooth muscle resting tension (Figure 1d). Interestingly, incubation of an individual colon preparation with the Fab fragment of $\operatorname{IgG}$ from a narcolepsy patient did not alter CMMC activity, and subsequent addition of neostigmine did not significantly alter the smooth muscle resting tension $(P=0.167)$, suggesting that cross-linking of the target by the Ab may be required for functional activity (Supplementary Figure 2).

\section{Anti-M3R Ab-Mediated Slowing of CMMCs is Reversed by Neostigmine}

As anti-M3R Abs from patients with SS have been reported to decrease the frequency of CMMCs, ${ }^{24}$ we tested whether the addition of neostigmine to the CMMC assay could distinguish a narcoleptic IgG-mediated decrease in CMMC frequency from that mediated by anti-M3R Abs. The addition of human IgG $(0.4 \mathrm{mg} / \mathrm{ml})$ with inhibitory anti-M3R Abs decreased the frequency of CMMCs (average pre-IgG CMMC frequency $112.4 \mathrm{~s}$, average post-IgG CMMC frequency $176.8 \mathrm{~s})$. Subsequent addition of $1 \mu \mathrm{mol} / \mathrm{l}$ neostigmine restored CMMC frequency without increasing smooth muscle tension (Figure 2a and c). By contrast, neostigmine failed to reverse narcoleptic IgG-mediated slowing of CMMCs, producing an increase in resting tension and phasic contractions (Figure $2 \mathrm{~b}$ and $\mathrm{c}$ ). Hence, neostigmine can distinguish narcoleptic IgG-mediated slowing of CMMCs from a reduction in frequency caused by anti-M3R Abs.

\section{Cholinergic Blockade with Atropine Reverses the Effects of Neostigmine on Narcoleptic IgG}

To determine whether neostigmine potentiates narcoleptic IgG-mediated colon smooth muscle hyperactivity through a cholinergic mechanism, we examined its reversibility by the anticholinergic drug, atropine. Neostigmine was first used to potentiate the narcoleptic IgG-mediated increase in smooth muscle resting tension. Subsequent addition of $1 \mu \mathrm{mol} / \mathrm{l}$ atropine to the colon preparation restored the smooth muscle resting tension to pre-neostigmine levels (Figure $3 \mathrm{a}$ and $\mathrm{b}$ ). These data show that neostigmine enhances narcoleptic IgG-mediated cholinergic hyperactivity in colonic smooth muscle.

\section{TTX Mimics the Effect of Narcoleptic IgG}

TTX, a blocker of voltage-gated sodium channels, has been reported by others to abolish CMMC activity and to increase smooth muscle resting tension in the isolated colon. ${ }^{23}$ Therefore, we investigated whether TTX could mimic the actions of the narcoleptic IgG. In colonic preparations undergoing CMMC activity, addition of $0.6 \mu \mathrm{mol} / 1 \mathrm{TTX}$ abolished CMMCs and increased smooth muscle resting tension (Figure 4a). Addition to the preparation of $1 \mu \mathrm{mol} / \mathrm{l}$ neostigmine significantly increased smooth muscle resting tension compared with TTX alone $(P<0.0001$, Wilcoxon matchedpairs comparison of pre- $v s$ post-drug mean contraction amplitude). As observed in the parallel experiments with narcoleptic IgG (Figure 3), atropine $(1 \mu \mathrm{mol} / \mathrm{l})$ reversed the neostigmine-mediated increase in smooth muscle tension (Figure $4 \mathrm{a}$ and $\mathrm{b}$ ). Thus, TTX, an ion channel blocker that binds both excitatory and inhibitory motor neurons in $\mathrm{CMMCs},{ }^{23}$ mimics the physiological effects of the narcoleptic IgG on the CMMC bioassay. 
a

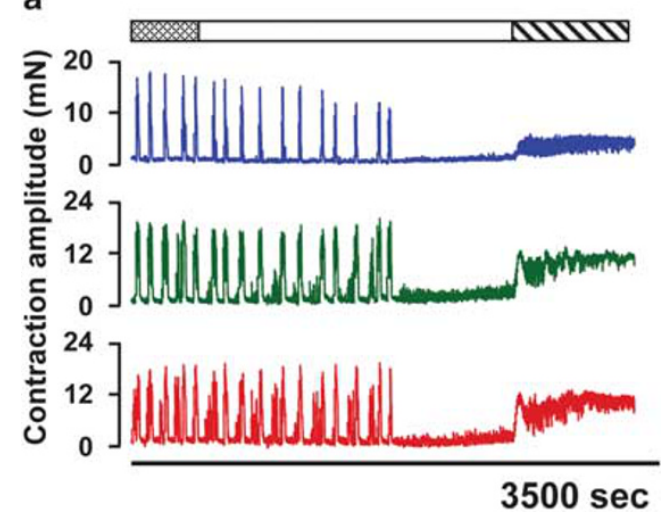

C

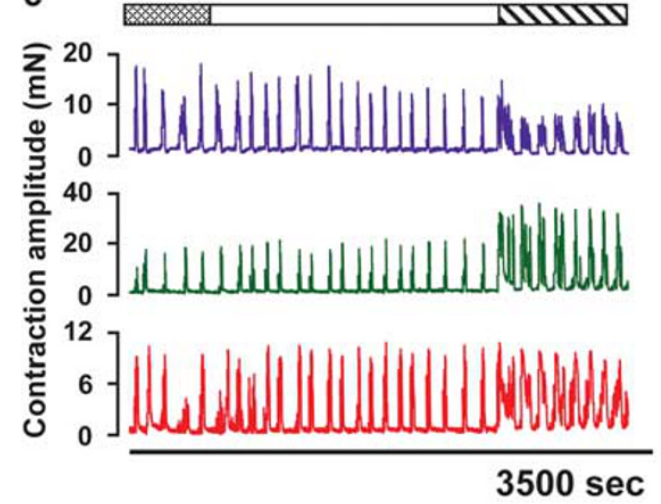

b
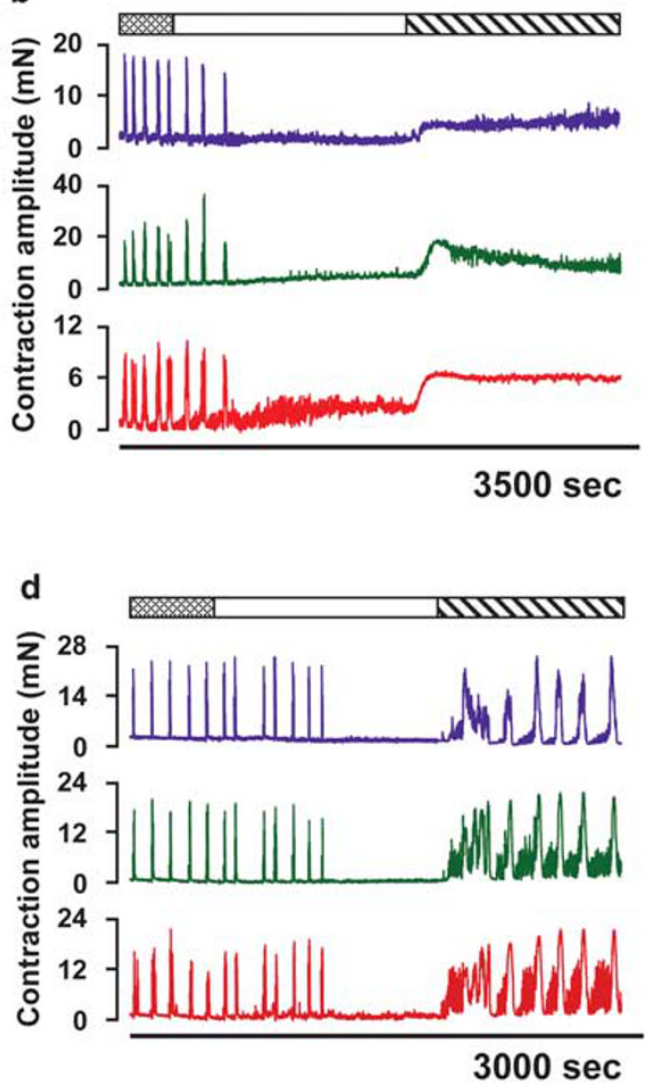

e



Figure 1 The cholinesterase inhibitor, neostigmine, enhances smooth muscle resting tension in the presence of IgG from patients with narcolepsycataplexy and fails to restore CMMC activity in the mouse isolated colon. Representative tracings of CMMC activity measured at three recording points along the colon from the proximal (top trace) to distal (bottom trace). The spontaneous, contractile CMMC activity occurs every 3-5 min, separated by periods of relative quiescence (smooth muscle resting tension). (a and $\mathbf{b})$ The normal pattern of CMMC activity (baseline, resting tension is increased by the addition of narcoleptic lgG $(0.4 \mathrm{mg} / \mathrm{ml})(\square)$. Addition of neostigmine $(1 \mu \mathrm{mol} / \mathrm{l})(\boldsymbol{\nabla})$ ) further increased smooth muscle resting tension and did not restore CMMC activity. (c) CMMC activity is unchanged by addition of lgG $(0.4 \mathrm{mg} / \mathrm{ml})$ from a normal control. Subsequent addition of neostigmine did not alter smooth muscle resting tension. (d) In experiments in which excess control lgG $(0.8 \mathrm{mg} / \mathrm{ml}) \mathrm{was}$ used to nonspecifically quench CMMCs, addition of neostigmine restored CMMC activity $(n=2)$. (e) Pooled smooth muscle resting tension data. Neostigmine caused a significant increase in resting tension in the presence of narcoleptic $\operatorname{lgG}(P<0.0001, n=6)$ but not with control $\operatorname{lgG}(n=8)$. Values are mean and s.e.m. 
a

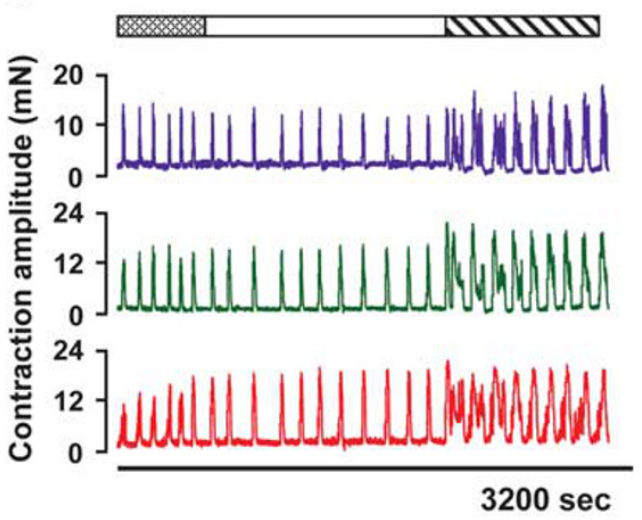

b

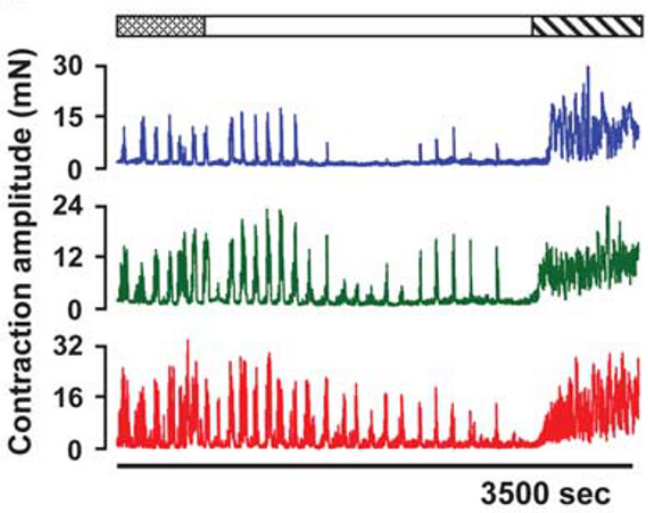

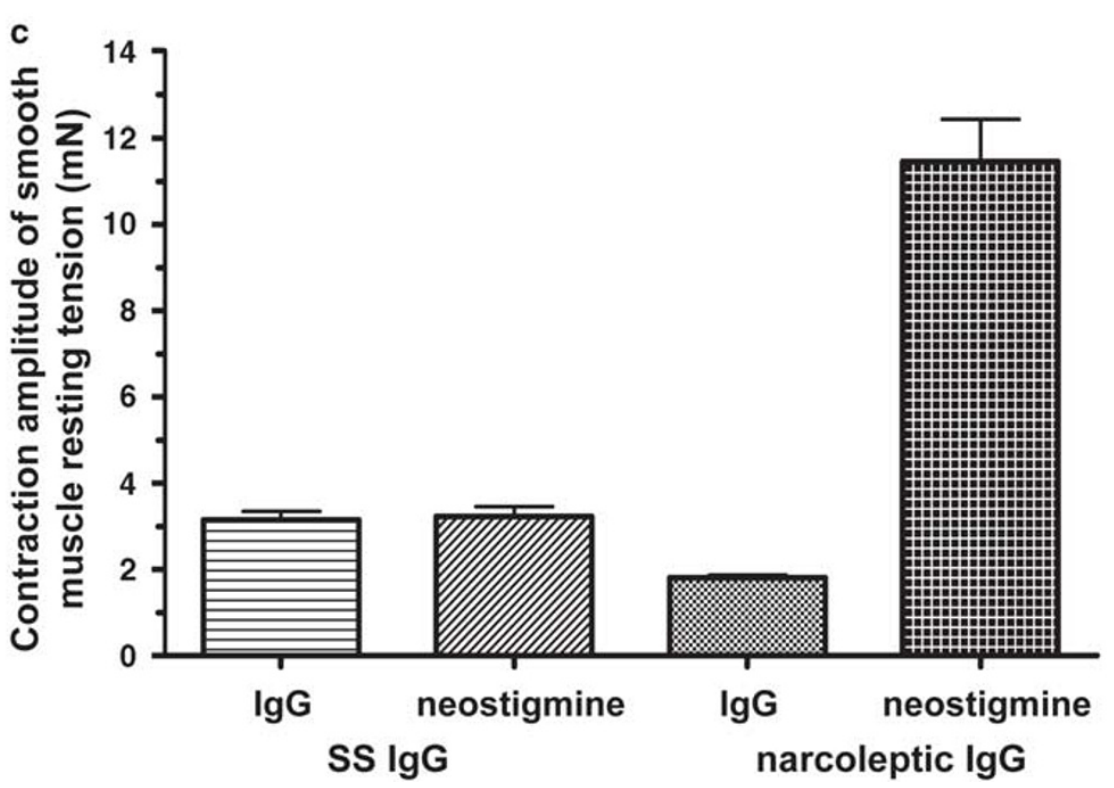

Figure 2 Challenge with neostigmine reverses anti-M3R Ab-mediated slowing of CMMCs and potentiates narcoleptic lgG. (a) Tracing of CMMC activity in a preparation incubated with IgG containing inhibitory anti-M3R Abs from a patient with primary SS. The frequency of CMMCs was decreased after the addition of the $\mathrm{lgG}(0.4 \mathrm{mg} / \mathrm{ml})$, and restored by addition of $1 \mu \mathrm{mol} / /$ neostigmine. (b) Representative tracing of CMMC activity in a preparation with narcoleptic lgG-mediated slowing of CMMCs. Neostigmine induced large amplitude, phasic contractions together with increased smooth muscle tension. (c) Pooled smooth muscle resting tension data. Neostigmine significantly increased smooth muscle resting tension $(P<0.0001)$ in preparations with narcoleptic IgG-mediated slowing of CMMCs $(n=2)$, but not in a preparation incubated with SS IgG. Values are mean and s.e.m.

\section{DISCUSSION}

Recently, we reported that narcoleptic IgG abrogated CMMC activity and increased smooth muscle resting tone. ${ }^{21}$ Excitation of intrinsic cholinergic motor neurons has been shown to have an essential role in the generation of CMMCs. ${ }^{27} \mathrm{In}$ contrast, in the interval between CMMCs, inhibitory motor neurons maintain the circular smooth muscle under tonic inhibition. ${ }^{23}$ We used the cholinesterase inhibitor, neostigmine, to further explore the mechanism of narcoleptic IgG-mediated disruption of enteric motor function. Neostigmine functions extracellularly in the neuromuscular junction by blocking the active site of acetylcholinesterase, thereby increasing the availability of acetylcholine at muscarinic receptors. In this study, neostigmine induced a sustained and significant increase in smooth muscle resting tone in preparations incubated with narcoleptic IgG, regardless of whether CMMC activity was abolished or partially retained. The neostigmine-induced enhancement of smooth muscle tension occurred specifically in the presence of the narcoleptic IgG, and not with control IgG or IgG with antiM3R activity. In addition, neostigmine restored CMMC activity to preparations in which the addition of an excess of control IgG resulted in the loss of CMMC activity, but not in preparations in which narcoleptic IgG had abolished CMMCs, indicating that a nonspecific loss of CMMC activity can be reversed by neostigmine. Accordingly, challenge with the drug neostigmine improves the stringency of the CMMC assay for the narcoleptic Ab. 
a

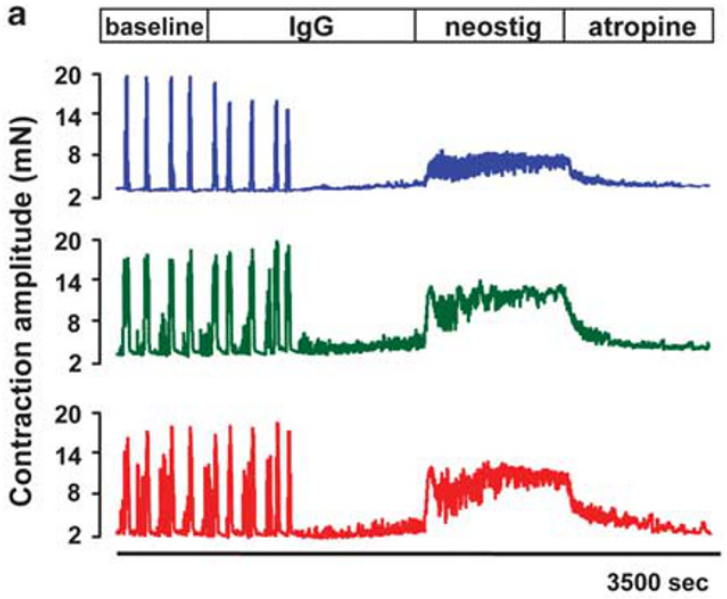

b

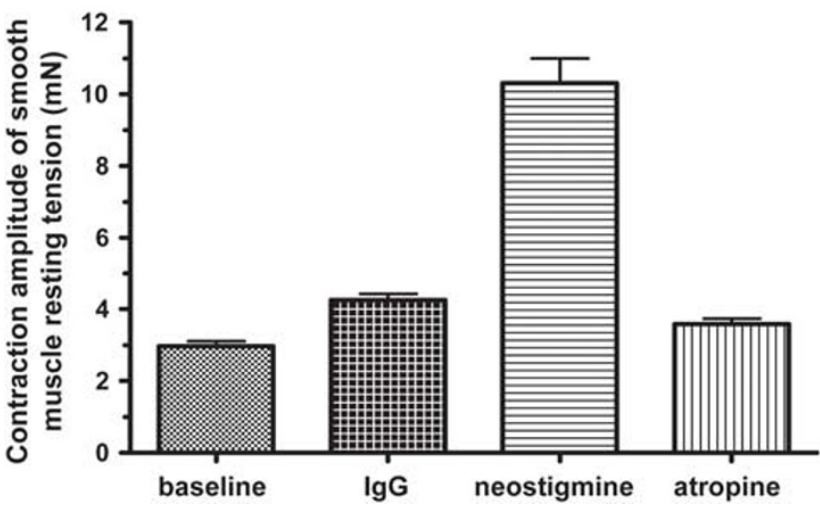

Figure 3 The neostigmine-mediated potentiation of narcoleptic IgG is reversed by atropine. (a) Representative tracing of a preparation with narcoleptic lgG-mediated loss of CMMCs and increased smooth muscle tension. Addition of neostigmine $(1 \mu \mathrm{mol} / \mathrm{l})$ further increased smooth muscle resting tension, which was reversed by atropine $(1 \mu \mathrm{mol} / \mathrm{l})$. (b) Pooled smooth muscle resting tension data. In preparations undergoing CMMC activity, addition of narcoleptic IgG mediates an increase in smooth muscle tension. Subsequent addition of neostigmine further increases resting tension, which is reversed by atropine $(n=4)$. Values represent the mean and s.e.m.

A key finding of this study was that neostigmine produced an atropine-sensitive enhancement of the narcoleptic IgGmediated increase in smooth muscle resting tension and phasic contractions. Although the possibility remains that multiple Abs are present in narcolepsy, neostigmine enhanced the narcoleptic IgG-mediated effect consistently in each subject, arguing for the presence of a single functional antigenic target. However, we cannot exclude the coexistence of stimulatory and inhibitory Abs against a common target, as has been described in patients with Grave's disease who may have stimulating and blocking Abs against the thyrotropin receptor. ${ }^{28}$ A direct effect of the narcoleptic Ab on muscarinic receptors expressed on the smooth muscle seems unlikely, as narcoleptic IgG did not alter carbachol responses of isolated smooth muscle strips from either the colon (data not shown) or bladder. ${ }^{29}$ Addition of neostigmine to the CMMC
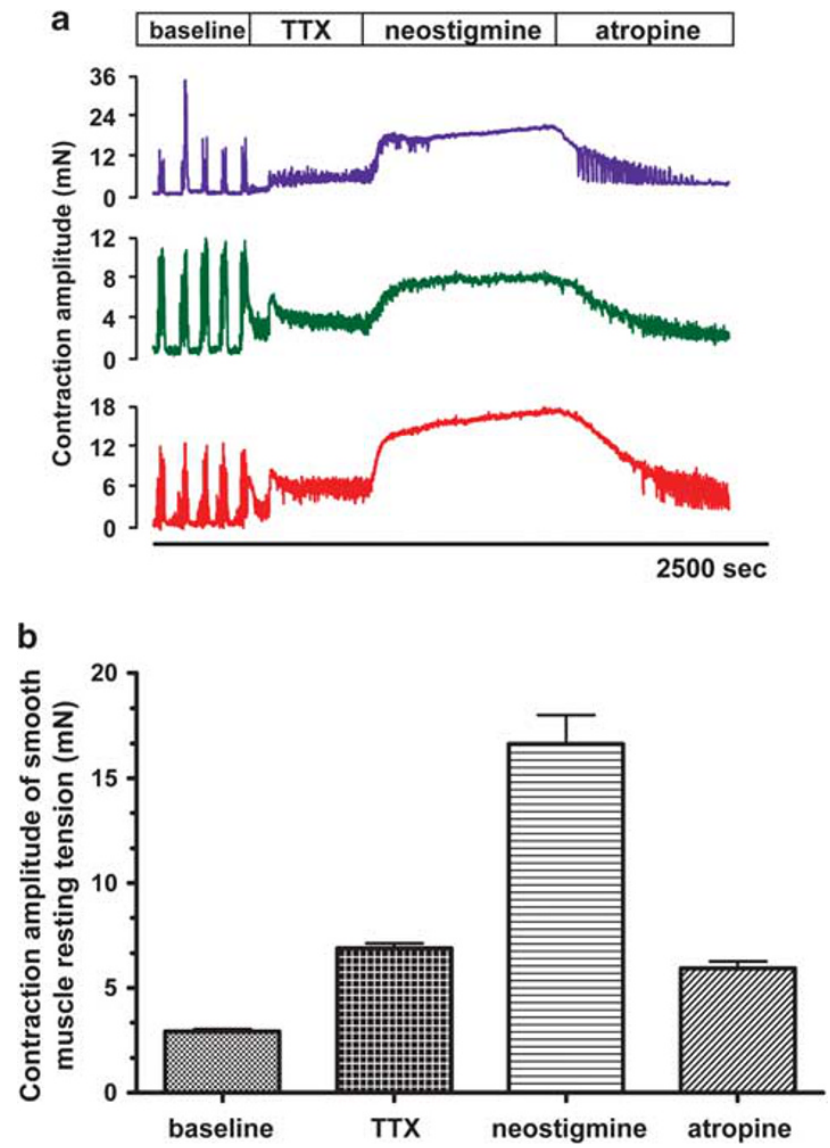

Figure $4 \pi T X$, a sodium channel blocker, is a mimic for the effects of narcoleptic IgG on the CMMC assay. (a) Representative tracing of CMMC activity in the presence of TTX and atropine. Normal CMMC activity is abolished and smooth muscle resting tension is increased by the addition of TTX $(0.6 \mu \mathrm{mol} / \mathrm{l})$. Smooth muscle resting tension was further increased by addition of neostigmine $(1 \mu \mathrm{mol} / \mathrm{l})$, and reversed by atropine $(1 \mu \mathrm{mol} / \mathrm{l})$. (b) Pooled smooth muscle resting tension data. The TTX-mediated increase in smooth muscle resting tension is potentiated by neostigmine and reversed by atropine $(n=3)$. Values represent the mean and s.e.m.

preparation caused a similar atropine-reversible potentiation of smooth muscle tension increases in the presence of TTX, showing the latter as a mimic of the functional effects of the narcoleptic Ab. TTX, a blocker of neuronal sodium channels, has been reported to prevent neurotransmission from both excitatory and inhibitory motor nerves, resulting in the loss of CMMCs and disinhibition of the smooth muscle. ${ }^{23,30}$ Significantly, disruption of excitatory ganglionic neurotransmission by the nicotinic receptor antagonist, hexamethonium, abolishes CMMCs but does not result in changes to smooth muscle resting tone, concluded to be due to residual inhibitory motor nerve activity, ${ }^{23}$ arguing against an effect of the narcoleptic $\mathrm{Ab}$ on nicotinic acetylcholine receptors. The most plausible scenario is that the narcoleptic $\mathrm{Ab}$, similar to its mimic TTX, exerts a functional blockade of excitatory and inhibitory enteric motor nerves by targeting a 
receptor or ion channel present on both classes of motor neurons. Interestingly, although the divalent $\mathrm{F}\left(\mathrm{ab}^{\prime}\right)_{2}$ fragment of narcoleptic IgG is active in the colon assay, ${ }^{21}$ the Fab fragment failed to exert an effect in CMMC activity, suggesting that cross-linking of the target by the $\mathrm{Ab}$ may be a requirement for functional activity. Identifying the specific target of the $\mathrm{Ab}$ is a critical area for future research and will presumably require the development of cell-based assays. Whole-organ physiological assays are unsuited for this task but will be a useful gold standard for new assay development. Flow cytometric analysis of cells overexpressing candidate autoantigens and patch clamping studies are two methodologies that are currently under consideration in our laboratory.

At first glance, it seems paradoxical that narcoleptic IgG and its TTX mimic can abolish CMMCs while at the same time enhance smooth muscle cholinergic activity. These findings suggest an ongoing availability of ACh in the presence of a blockade of excitatory motor nerves. A clue comes from studies of the neuromuscular junction of rat diaphragm that show a spontaneous leakage of ACh from excitatory motor nerves in the absence of neuronal stimulation. In this model, neuronal ACh release seems to come predominantly from cytoplasmic stores, rather than from secretory vesicles. ${ }^{31}$ More recently, efferent nerve endings in visceral organs have been shown to release ACh in the presence of TTX. $^{32}$ We suggest that an ongoing release of cytoplasmic ACh from enteric motor neurons in colonic smooth muscle facilitates cholinergic responses in the absence of excitatory motor nerve activity. In the presence of narcoleptic IgG or its TTX mimic, neostigmine potentiates smooth muscle responses to ACh released from inactive excitatory motor neurons.

In summary, the cholinesterase inhibitor, neostigmine, dramatically potentiates narcoleptic IgG-mediated smooth muscle cholinergic hyperactivity in an isolated whole colon preparation. We propose a model of Ab-mediated disruption of enteric neural function, whereby a functional $\mathrm{Ab}$ in narcolepsy alters the regulation of colonic motility by exerting a blockade of both excitatory and inhibitory motor neurons, resulting in a loss of CMMCs and the induction of cholinergic hyperactivity in the disinhibited smooth muscle (Figure 5). As functional Abs have been described as mediating either excitatory or inhibitory actions on their targets, ${ }^{18}$ the putative narcoleptic $\mathrm{Ab}$ could conceivably act by either activating or inhibiting an ion channel common to both classes of enteric motor nerves. The relationship of these findings to the pathophysiology of human narcolepsy remains unresolved, as there is no apparent link to an autoimmune process leading to the destruction of hypocretin-producing neurons that causes narcolepsy. However, narcoleptic IgG-mediated cholinergic hypersensitivity, and its potentiation by neostigmine, has intriguing similarities with earlier pharmacological studies of canine narcolepsy, in which the threshold for carbachol-induced cataplexy is

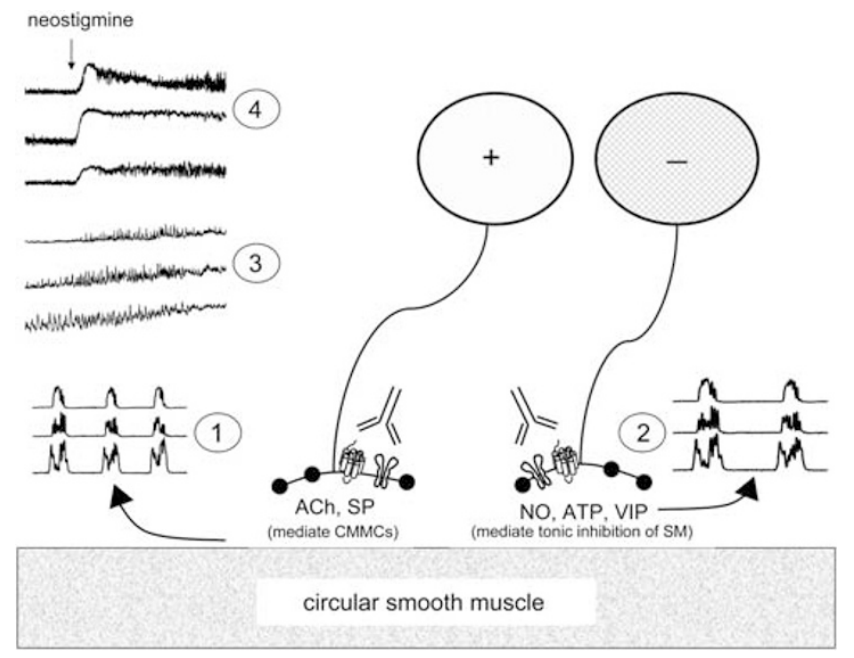

Figure $5 \mathrm{~A}$ model for CMMC disruption by a functional autoantibody in narcolepsy. The periodic release of neurotransmitters from excitatory motor neurons results in the formation of CMMCs (1). Between CMMCs, inhibitory motor neurons maintain the circular smooth muscle under tonic inhibition (2). The narcoleptic Ab binds to a receptor or ion channel expressed on both excitatory and inhibitory motor neurons, resulting in a functional blockade of enteric motor nerve activity, with the loss of CMMCs. Ongoing leakage of cytoplasmic ACh from excitatory motor nerves results in an increase in resting tension and cholinergic activity in the disinhibited circular smooth muscle (3). The cholinesterase inhibitor, neostigmine, potentiates the narcoleptic IgG-mediated cholinergic activity and smooth muscle resting tension, without restoring CMMCs (4). ACh, acetylcholine; ATP, adenosine triphosphate; CMMCs, colonic migrating motor complexes; NO, nitric oxide; SM, smooth muscle; SP, substance P; VIP, vasoactive intestinal peptide.

reduced and systemic administration of a cholinesterase inhibitor increases canine cataplexy reducible by atropine. ${ }^{33-35}$ We suggest that additional pharmacological and genetic dissection of the surrogate CMMC assay offers a useful entry point for further study of the functional narcoleptic $\mathrm{Ab}$.

Supplementary Information accompanies the paper on the Laboratory Investigation website (http://www.laboratoryinvestigation.org)

\section{ACKNOWLEDGEMENTS}

We thank Dr Doug McEvoy and Dr Nick Antic for assistance in recruiting the patients and Dr Pamela McCombe for her critical comments on the paper. This study was supported by a project Grant no. 375106 from the National Health and Medical Research Council of Australia.

\section{DISCLOSURE/CONFLICT OF INTEREST}

The authors declare no conflict of interest.

1. Overeem S, Mignot E, van Dijk JG, et al. Narcolepsy: clinical features, new pathophysiologic insights, and future perspectives. J Clin Neurophysiol 2001;18:78-105.

2. Scammell TE. The neurobiology, diagnosis, and treatment of narcolepsy. Ann Neurol 2003;53:154-166.

3. Nishino S, Ripley B, Overeem S, et al. Hypocretin (orexin) deficiency in human narcolepsy. Lancet 2000;355:39-40. 
4. Peyron C, Faraco J, Rogers W, et al. A mutation in a case of early onset narcolepsy and a generalized absence of hypocretin peptides in human narcoleptic brains. Nat Med 2000;6:991-997.

5. Thannickal TC, Moore RY, Nienhuis R, et al. Reduced number of hypocretin neurons in human narcolepsy. Neuron 2000;27:469-474.

6. Rogers $A E$, Meehan J, Guilleminault C, et al. HLA DR15 (DR2) and DQB1*0602 typing studies in 188 narcoleptic patients with cataplexy. Neurology 1997;48:1550-1556.

7. Mignot E, Lin L, Rogers W, et al. Complex HLA-DR and -DQ interactions confer risk of narcolepsy-cataplexy in three ethnic groups. Am J Hum Genet 2001;68:686-699.

8. Hallmayer J, Faraco J, Lin L, et al. Narcolepsy is strongly associated with the T-cell receptor alpha locus. Nature Genetics print copy in press May 2009. Available from: URL: www.nature.com/ng/journal/vaop/ ncurrent/abs/ng.372.html.

9. Black III JL. Narcolepsy: a review of evidence for autoimmune diathesis Int Rev Psychiatry 2005;17:461-469.

10. Scammell TE. The frustrating and mostly fruitless search for an autoimmune cause of narcolepsy. Sleep 2006;29:601-602.

11. Overeem S, Black III JL, Lammers GJ. Narcolepsy: immunological aspects. Sleep Med Rev 2008;12:95-107.

12. Honda M, Eriksson KS, Zhang $\mathrm{S}$, et al. IGFBP3 colocalizes with and regulates hypocretin (orexin). PLoS ONE 2009;4:e4254.

13. Miyagawa T, Kawashima M, Nishida N, et al. Variant between CPT1B and $\mathrm{CHKB}$ associated with susceptibility to narcolepsy. Nat Genet 2008;40:1324-1328.

14. Nishino S, Mignot E. Pharmacological aspects of human and canine narcolepsy. Prog Neurobiol 1997;52:27-78.

15. Guilleminault $\mathrm{C}$, Heinzer R, Mignot $\mathrm{E}$, et al. Investigations into the neurologic basis of narcolepsy. Neurology 1998;50:S8-S15.

16. Jackson MW, Gordon TP, Waterman SA. Disruption of intestinal motility by a calcium channel-stimulating autoantibody in type 1 diabetes. Gastroenterology 2004;126:819-828.

17. Wang F, Jackson MW, Maughan V, et al. Passive transfer of Sjogren's syndrome lgG produces the pathophysiology of overactive bladder. Arthritis Rheum 2004;50:3637-3645.

18. Waterman SA, Jackson MW, Gordon TP. Functional effects of autoantibodies. In: Mackay IR, Rose NR (eds). The Autoimmune Diseases, 4th edn. Elsevier: Sydney, 2006, pp 217-236.

19. Wan EC, Gordon TP, Jackson MW. Autoantibody-mediated bladder dysfunction in type 1 diabetes. Scand J Immunol 2007;65:70-75.

20. Wan EC, Gordon TP, Jackson MW. Autoantibodies to calcium channels in type 1 diabetes mediate autonomic dysfunction by different mechanisms in colon and bladder and are neutralized by antiidiotypic antibodies. J Autoimmun 2008;31:166-172.

21. Jackson MW, Reed JH, Smith AJ, et al. An autoantibody in narcolepsy disrupts colonic migrating motor complexes. J Neurosci 2008;28:13303-13309.

22. Cestele S, Catterall WA. Molecular mechanisms of neurotoxin action on voltage-gated sodium channels. Biochimie 2000;82:883-892.

23. Fida R, Lyster DJ, Bywater RA, et al. Colonic migrating motor complexes (CMMCs) in the isolated mouse colon. Neurogastroenterol Motil 1997;9:99-107.

24. Park K, Gordon TP, Jackson MW. Functional autoantibodies in primary Sjogren's syndrome disrupt gastrointestinal tract motility by targeting the muscarinic 3 receptor. American College of Rheumatology. Arthritis Rheum, San Francisco 2008, pp S795.

25. Steinbuch $M$, Audran R. The isolation of IgG from mammalian sera with the aid of caprylic acid. Arch Biochem Biophys 1969;134:279-284.

26. Cavill D, Waterman SA, Gordon TP. Antiidiotypic antibodies neutralize autoantibodies that inhibit cholinergic neurotransmission. Arthritis Rheum 2003;48:3597-3602.

27. Spencer NJ, Hennig GW, Dickson E, et al. Synchronization of enteric neuronal firing during the murine colonic MMC. J Physiol 2005;564:829-847.

28. Kohn LD, Harli H. Thyrotropin receptor autoantibodies (TSHRAbs): epitopes, origins and clinical significance. Autoimmunity 2003;36: 331-337.

29. Smith AJ, Jackson MW, Neufing $P$, et al. A functional autoantibody in narcolepsy. Lancet 2004;364:2122-2124.

30. Spencer NJ. Control of migrating motor activity in the colon. Curr Opin Pharmacol 2001;1:604-610.

31. Smith DO. Acetylcholine storage, release and leakage at the neuromuscular junction of mature adult and aged rats. J Physiol 1984;347:161-176.

32. Zagorodnyuk V, Gregory S, Costa M, et al. Spontaneous release of acetylcholine from autonomic nerves in the bladder. $\mathrm{Br} J$ Pharmacol 2009;157:607-619.

33. Delashaw Jr JB, Foutz AS, Guilleminault C, et al. Cholinergic mechanisms and cataplexy in dogs. Exp Neurol 1979;66:745-757.

34. Nishino S, Tafti M, Reid MS, et al. Muscle atonia is triggered by cholinergic stimulation of the basal forebrain: implication for the pathophysiology of canine narcolepsy. J Neurosci 1995;15:4806-4814.

35. Reid MS, Tafti M, Geary JN, et al. Cholinergic mechanisms in canine narcolepsy-I. Modulation of cataplexy via local drug administration into the pontine reticular formation. Neuroscience 1994;59:511-522. 